\title{
Detecting coral bleaching, using QuickBird multi-temporal data: A feasibility study at Kish Island, the Persian Gulf.
}

\begin{abstract}
Coral bleaching events have become more frequent and intense worldwide and speculated to be a severe threat for coral survival in future. The Persian Gulf, as one of the warmest seas, has also experienced coral mortalities and bleaching events. Historically, bleaching events are known to occur south of the Persian Gulf, such information is scarce in the northern side. Perhaps remoteness and inaccessibility to Iran main coral communities which have developed on offshore islands can explain such lack of data. To address this issue, the feasibility of using multi-temporal satellite images for detecting past bleaching events were investigated. Two QuickBird images $(2005,2008)$ were selected to detect 2007 bleaching event at Kish Island, Iran, and the accuracy of results were compared to in situ observations. Current study might represent "algae-challenged" scenario in terms of having 7 months' time lapse between bleaching event and post-bleaching satellite image. As a result of this, we had algae-covered corals instead of white bleached corals. In the proposed procedure pre and post-bleaching images were classified, and changes in reflectance values within coral classes were interpreted as bleaching areas. By using this method we could eliminate the effect of missclassification between bleached corals and sand; as well as algae-covered corals and live corals. Furthermore, it is not necessary to have a post-bleaching image acquired during bleaching events, although having such image will increase the accuracy. The proposed technique detected $\sim 28 \%$ of bleached corals and the results support the idea that coral bleaching can be distinguished by detecting the changes in reflectance values in pre and postbleaching images. Understanding the occurrence, severity, and extent of past bleaching events may help us understand the population dynamics of Iran corals and reveal coral connectivity patterns in the Persian Gulf.
\end{abstract}

Item Article
Type:

Keyword: Coral reefs; Bleaching; Multi-temporal satellite images; Remote sensing; Persian Gulf; Kish Island 